\title{
PROCESSO DE ESTERILIZAÇÃO SOB A ÓTICA DOS PROFISSIONAIS DO CENTRO DE MATERIAL E ESTERILIZAÇÃO
}

\author{
Sterilization process from the perspective of the professionals of the material and sterilization center
}

\section{Procedimiento de esterilización bajo la óptica de los profesionales del centro de material y esterilización}

\author{
Lívia Maria Correia de Morais*, Solange Queiroga Serrano², Alessandra Nogueira Santos³, \\ Jéssica Maricelly Deodato de Oliveira $a^{4}$ Jéssica Thamires da Silva Melo ${ }^{5}$
}

RESUMO: Objetivo: Analisar o processo de trabalho dos profissionais de enfermagem atuantes no centro de material e esterilização (CME) acerca da esterilização de material cirúrgico. Método: Estudo descritivo, ancorado pela abordagem qualitativa e realizado com 11 profissionais de enfermagem do CME por meio de entrevista semiestruturada. Para avaliação dos dados, optou-se pela análise de conteúdo, modalidade temática transversal. Resultados: Os depoimentos apresentaram a descrição das etapas envolvidas no processo de esterilização, a relação com a segurança do paciente, as dificuldades no processo de trabalho e a educação permanente em saúde como meio possível de superar as dificuldades. Conclusão: Os trabalhadores têm conhecimento incipiente das etapas do processo de esterilização, com influência direta na segurança do paciente. Ações de gestão e de educação permanente em saúde são necessárias para garantir a qualidade do trabalho e possibilitar a redução de falhas nesse processo. Foram citadas, ainda, as dificuldades diárias encontradas pelo pessoal de enfermagem do CME. Palavras-chave: Infecção hospitalar. Esterilização. Segurança do paciente. Prática profissional. Educação continuada.

ABSTRACT: Objective: To analyze the work process of nursing professionals working in the material and sterilization center (MSC) on the sterilization of surgical material. Method: A descriptive study, anchored by qualitative approach and carried out with 11 nursing professionals from MSC, through a semi-structured interview. In order to evaluate the data, content analysis was chosen, with transversal thematic modality. Results: The interviews presented the description of the steps involved in the sterilization process, the relationship with patient safety, difficulties in the work process, and permanent health education as a possible mean of overcoming difficulties. Conclusion: Workers have incipient knowledge of the steps of the sterilization process with a direct influence on patient safety. Management actions and permanent education in health are necessary to guarantee the quality of work and to enable the reduction of failures in the work process. The daily difficulties encountered by MSC nursing staff were also related.

Keywords: Nosocomial infection. Sterilization. Patient safety. Professional practice. Continuing education.

RESUMEN: Objetivo: Analizar el proceso de trabajo de los profesionales de enfermería actuantes en el CME sobre la esterilización de material quirúrgico. Método: Estudio descriptivo, anclado por el abordaje cualitativo y realizado con 11 profesionales de enfermería del CME, por medio de entrevista semiestructurada. Para la evaluación de los datos, se optó por el análisis de contenido, modalidad temática transversal. Resultados: Los testimonios presentaron la descripción de las etapas involucradas en el proceso de esterilización, la relación con la seguridad del paciente, las dificultades en el proceso de trabajo y la educación permanente en salud como medio posible de superar las dificultades. Conclusión: Los trabajadores tienen conocimiento incipiente de las etapas del proceso de esterilización con influencia directa en la seguridad del paciente. Las acciones de gestión y de educación permanente en salud son necesarias para garantizar la calidad del mismo y posibilitar la reducción de fallas en el proceso de trabajo. Aún se relacionó las dificultades diarias encontradas por el personal de enfermería del CME.

Palabras clave: Infección hospitalaria. Esterilización. Seguridad del paciente. Práctica profesional. Educación continua.

Enfermeira especialista em Enfermagem Cirúrgica pelo Centro Acadêmico de Vitória, da Universidade Federal de Pernambuco - Recife (PE), Brasil.

${ }^{2}$ Enfermeira doutora. Professora adjunta e coordenadora das disciplinas de Enfermagem Cirúrgica e de Metodologia da Pesquisa do Centro Acadêmico de Vitória da Universidade Federal de Pernambuco. Tutora do Programa de Residência de Enfermagem Cirúrgica do Hospital Getúlio Vargas da Universidade Federal de Pernambuco - Recife (PE), Brasil.

${ }^{3}$ Enfermeira pela Universidade de Pernambuco; enfermeira especialista em Enfermagem Cirúrgica pelo Centro Acadêmico de Vitória da Universidade Federal de Pernambuco - Recife (PE), Brasil.

${ }^{4}$ Enfermeira e especialista em Enfermagem Cirúrgica pelo Centro Acadêmico de Vitória da Universidade Federal de Pernambuco - Recife (PE), Brasil.

${ }^{5}$ Enfermeira pela Universidade de Pernambuco; especialista em Ortopedia e Traumatologia pelo Centro Acadêmico de Vitória da Universidade Federal de Pernambuco - Recife (PE), Brasil.

*Autor correspondente: liecorreia@gmail.com

Recebido: 16/06/2017 - Aprovado: 07/04/2018

DOI: $10.5327 / Z 1414-4425201800020002$ 


\section{INTRODUÇÃO}

O centro de material e esterilização (CME) é uma unidade de apoio técnico destinada ao fornecimento de produtos para saúde (PPS) apropriadamente processados, para assistência à saúde dos indivíduos. Esse processo inclui: a limpeza, o preparo, a esterilização, o armazenamento e a distribuição dos materiais às demais áreas hospitalares ${ }^{1,2}$.

O cuidado direto ao paciente relaciona-se ao indireto, prestado pelos profissionais do CME, que devem fornecer suporte para a assistência, uma vez que a eficiência da esterilização possibilita a redução de infecções exógenas e a melhoria na qualidade do cuidado dispensado ao paciente ${ }^{3,4}$.

A compreensão sobre a influência dos procedimentos de limpeza, desinfecção e esterilização dos materiais na prevenção e no controle das infecções hospitalares ressalta a importância e a responsabilidade do CME no âmbito das instituições de saúde, pois a existência de falhas nesses processos é determinante ao surgimento de complicações nos pacientes 5 .

Historicamente, as atividades desenvolvidas no CME eram executadas por pessoas sem qualificação específica para exercer as complexidades do processamento dos materiais para saúde. A falta de programas de capacitação e atualização permanente para os profissionais, tanto para os que desempenham diretamente as ações quanto para os que utilizam os materiais esterilizados, prejudica a segurança da assistência pela falha de conhecimento das etapas do processamento ${ }^{4}$.

Essa realidade pode ser modificada ao se associar a qualificação dos profissionais ao desenvolvimento de suas atribuições com excelência ${ }^{6}$.

\section{OBJETIVO}

Identificar a percepção de profissionais de enfermagem atuantes no CME sobre o processo de trabalho na esterilização de material cirúrgico.

\section{MÉTODO}

O estudo fundamentou-se na abordagem qualitativa, de natureza descritiva e exploratória, que enfatiza as singularidades e o significado apreendidos pelos profissionais da enfermagem acerca do processo de trabalho no CME pertinentes ao fenômeno estudado ${ }^{7}$.
Para tanto, o estudo foi realizado no CME de um hospital público do Recife (PE) que é referência nas áreas de cirurgias de tráumato-ortopedia e cirurgias gerais, com enfermeiros, técnicos e auxiliares de enfermagem atuantes nessa unidade. O critério de inclusão relaciona-se ao exercício das funções, no mínimo, por um ano no referido setor. Não foram incluídos os profissionais que estavam em licença-saúde, licença-maternidade ou férias.

Obedeceu-se aos critérios de saturação, em que não há novas ideias nos depoimentos, para totalizar a amostra, de 11 indivíduos. Os dados foram coletados no período de outubro de 2016 a fevereiro de 2017 por meio de entrevista semiestruturada em ambiente reservado, para alcançar a confidencialidade das informações obtidas.

Um instrumento foi elaborado pelas pesquisadoras, constituído de informações sociodemográficas dos participantes, tais como: idade, sexo, jornada de trabalho, tempo de serviço e escolaridade, para caracterização da amostra, e pelas seguintes questões:

1. O que você entende pelo processo de esterilização dos artigos críticos?

2. Como você relaciona o processamento de material no CME com a segurança do paciente durante sua prática profissional?

3. Quais são as dificuldades encontradas no seu cotidiano de trabalho?

4. Você recebe algum tipo de capacitação para realizar o seu trabalho? Como é feito esse processo?

As entrevistas foram gravadas em aparelho digital e, em seguida, transcritas na íntegra e duraram, em média, 7 minutos. Para assegurar o anonimato dos sujeitos, eles foram identificados pela letra E (Entrevistado), acompanhada da numeração da ordem da realização da entrevista (1, 2, 3 etc.) e de sua categoria funcional (E: enfermeiro; TE: técnico em enfermagem; AE: auxiliar de enfermagem).

A avaliação do material analítico efetuou-se pela análise de conteúdo; modalidade temática transversal que consta de pré-análise, exploração do material e tratamento dos resultados ${ }^{8}$. Na pré-análise, organizou-se, de acordo com o objetivo da pesquisa e a literatura, a ordenação das ideias iniciais ${ }^{8}$.

Na exploração do material, definiu-se o sistema de codificação pela identificação das unidades de registro ou núcleos dos sentidos e pela determinação das categorias. Depois, houve o tratamento dos resultados, com inferência e interpretação para condensar e destacar as informações 
coletadas, além de compará-las com a literatura pertinente ao assunto ${ }^{8}$.

O projeto foi aprovado pelo Comitê de Ética em Pesquisa do Hospital Otávio de Freitas sob o Certificado de Apresentação ara Apreciação Ética (CAEE) 58035316.0.0000.5200. Todos os participantes assinaram o Termo de Consentimento Livre e Esclarecido.

\section{RESULTADOS}

Dos 11 participantes, dez são do sexo feminino. São eles: dois enfermeiros, sete técnicos e dois auxiliares de enfermagem, com média de idade de 50 anos, tempo médio de serviço no setor igual a nove anos e média de 22,6 anos de formação profissional. A jornada de trabalho constitui-se de plantão de 12 horas por 60 horas de folga, e apenas um dos entrevistados era diarista, de 6 horas. Os dois participantes de nível superior possuem pós-graduação específica do setor.

Do total, quatro têm outro vínculo empregatício. Não foram identificados possíveis prejuízos em seu processo de trabalho por não mencionarem queixas de problemas relacionados à dupla jornada, como cansaço ou atrasos, entre outros.

A codificação dos depoimentos resultou no surgimento de quatro categorias, que serão descritas a seguir.

\section{Conhecimento dos profissionais sobre processamento de produtos para saúde}

O conhecimento dos profissionais acerca dos procedimentos realizados para esterilização de PPS foi descrito de forma sucinta, restringindo-se ao processo de limpeza e preparo do material:

Pega o material, coloca ele na solução enzimática; dá o tempo da gente tirar o material; a gente coloca embaixo da torneira, escova o material, tira a sujidade e leva para a sala de preparo. Coloca na máquina de secagem (E10, TE).

O material passa pela lavagem, vai para máquina de secar, segue para a mesa de arrumação e daí vai ser embalado (E8, TE).

Ser lavado, depois na mesa, no processo de secagem, para ir ser embalado (E6, AE).
Quanto à etapa da limpeza, apenas um dos participantes fez referência ao tempo de imersão dos produtos em soluções, porém de forma duvidosa e incorreta:

É feita a lavagem com soluções adequadas. Nessa, o pessoal prepara e mergulha. O material tem que ficar submerso por um tempo. Não lembro muito bem se são 10 ou 15 minutos (E5, $\mathrm{AE})$.

A investigação criteriosa da limpeza é um dos pontos críticos para que um produto seja reutilizado. Em relação ao preparo dos materiais, grande parte dos depoimentos descreveu o processo, apesar de não mencionar sua inspeção:

Na mesa de preparo o produto é colocado na secadora. É separado pelo tipo de peça, e ele vai para mesa de embalagem (E4, TE).

Passa pela secadora, quando o material chega na mesa, já chega sequinho. Então, só é arrumar, colocar nas caixas (E7, TE).

\section{Importância do processamento de materiais na segurança do paciente}

As atividades desenvolvidas no CME apresentam forte interferência no produto final durante a assistência. A unanimidade nas respostas demonstrou a relevância de o processamento ser realizado de forma correta, para evitar possíveis complicações, como mostra o trecho a seguir:

Se tiver alguma falha em um desses processos, eu vou estar emitindo risco para o paciente com relação à contaminação e às infecções (E11, E).

Você tem que entregar o material totalmente estéril, para não prejudicar a saúde do paciente. E tem que estar isento de qualquer infecção (E2, TE).

Para evitar a infecção. Porque o material necessita ser esterilizado, uma vez que ele vai ter contato direto com o paciente (E3, TE).

Um dos participantes salientou a importância de avaliar a efetividade do processo de esterilização por meio de indicadores: 
Para segurança do paciente, ele tem que ter, dependendo do material, uma fita, um indicador químico que vai dentro deste material indicando todo processo que passa na esterilização. Se houver uma falha neste indicador, significa que o material não está bem esterilizado. Então o objetivo é dar melhores condições de cirurgia ao paciente, evitando, assim, uma infecção, seja ela qual for (E6, AE).

\section{Dificuldades encontradas no processo de trabalho no centro de material e esterilização}

Os entrevistados expuseram as dificuldades encontradas na rotina de trabalho, apontando a falta de materiais fundamentais e de equipamentos de proteção individual (EPIs) como responsável pelo mau funcionamento do setor:

Tem dia que falta grau cirúrgico; tem dia que faltam faixas de vários tamanhos, aí eu vejo a necessidade de materiais [reposição] (E8, TE).

Com relação ao quantitativo de material, a gente precisaria ter mais instrumental para estar repondo $(\mathrm{E} 11, \mathrm{E})$.

A falta de material é devido a ser um hospital público, que está em crise e depende de licitação. Então, tem essa problemática (E1, E).

A dificuldade é [a falta de] material de proteção para nós, por exemplo. No meu caso, é a falta de óculos, uma viseira, entendeu? A falta de uma luva adequada para se pegar o material quente (E6, AE).

A falta de coisas [material] aqui é luva, luva é o principal (E10, TE).

Por outro lado, percebeu-se resistência dos profissionais quanto ao uso dos EPIs, como ressaltado em uma das falas sobre as dificuldades no cotidiano de trabalho:

É a proteção do funcionário e o uso dos EPIs. Eles têm muita resistência em usar (E1, E).

Outro fator que interfere na qualidade dos serviços de esterilização é a quantidade insuficiente de trabalhadores existentes, além da lotação de profissionais de enfermagem com problemas físicos ou psicológicos, o que, pelos depoimentos, é frequente e inadequado para a demanda do setor:

Déficit de pessoal, a gente precisa justamente ter um quadro maior de funcionários para que a gente possa estar melhorando a qualidade do serviço (E11, E).

Agora falta gente, é muito serviço para pouco pessoal; a mão de obra falta (E2, TE).

Na maioria das vezes, o CME é muito mal visto, porque é o local que vai as pessoas mais velhas, vai as pessoas doentes, que não prestam para nada ( $\mathrm{E} 3, \mathrm{TE})$.

Quando tem problema de saúde, a visão deles [gestores] é que aqui é o lugar, mas não é, porque a gente sabe que aqui fazemos muita coisa, e depende muito também do esforço físico, é muita coisa pesada (E1, E).

\section{Educação permanente dos profissionais como estratégia de melhoria no serviço}

Na práxis dos profissionais do CME, a falta de capacitação é um limitante na superação das dificuldades diárias:

Me passaram para eu ir treinando com as colegas mesmo. As que têm mais tempo foram me ensinando, aí eu fui aprendendo (E8, TE).

Então, como tem coisa muito específica, é uma área muito vasta, eu sinto essa dificuldade, porque eu tive que aprender com a cara. Cada dia você aprende uma coisa diferente, e eu acho que teria a necessidade de ter esse processo de reciclagem com os profissionais (E3, TE).

De acordo com as narrativas, quando ocorriam cursos da educação permanente associados à remuneração extra nos proventos, os trabalhadores sentiam-se estimulados a participar, sendo esse aspecto da instituição considerado positivo:

O objetivo do hospital é estar capacitando o profissional como um todo, até por conta da questão de 
cargos e carreiras, que tem um aumento na produtividade em cima de cada oito horas de curso semestral que a gente faz. Então, é uma forma de incentivar os funcionários a estar se qualificando (E3, TE).

Inclusive tem a produtividade. Sem essa produtividade, com curso é tanto, sem curso vai ser pior. Então, você obrigatoriamente tem que bus$\operatorname{car}(\mathrm{E} 2, \mathrm{TE})$.

Do mesmo modo, a despeito da importância do enfermeiro como gerenciador dessas atividades, apenas um dos respondentes destacou o papel do enfermeiro como educador:

A enfermeira que orienta a gente ensina através das informações que a gente tem ali na literatura, naqueles livros que mostra a figura e mostra $o$ nome dos instrumentais (E9, TE).

\section{DISCUSSÃO}

O CME é o setor responsável por concentrar os PPS esterilizados ou não, conservando-os e mantendo-os em boas condições. Para o desenvolvimento adequado de sua dinâmica de funcionamento e o efetivo processamento do material, é ideal que este seja submetido às seguintes etapas, de forma sequencial: recepção, limpeza/desinfecção, preparo, esterilização, armazenamento e distribuição ${ }^{1}$.

Pesquisa realizada em um hospital público de Porto Alegre (RS) com profissionais de enfermagem atuantes no CME mostrou que a maioria dos profissionais descreve o processo da limpeza e preparação dos materiais de forma concisa, o que pode ser justificado pela realização de atividades práticas isoladas, sem articulação teórica, tornando os funcionários meros executores de tarefas, mecanizados ${ }^{3,9}$.

Quanto maior a dificuldade na etapa da limpeza, maior será a dificuldade na da esterilização, considerando que a limpeza é o núcleo central do processamento de artigos. Assim, quando não é possível garantir a segurança no preparo, não se deve proceder à esterilização do material. Dúvidas, falta de conhecimento e técnicas inadequadas na lavagem podem implicar falhas no reprocessamento dos instrumentos ${ }^{3}$.

Após o recebimento do material, este deve ser totalmente submerso em solução contendo detergente enzimático, em temperatura morna, por no mínimo 3 minutos, ou como orientado pelo fabricante ${ }^{3,10}$. Apesar da importância do tempo de imersão, apenas um dos participantes fez referência a essa questão, mas de maneira duvidosa e incompleta. Tal fato poderia estar relacionado ao não uso de protocolos no serviço, na capacitação da equipe e/ ou na supervisão do enfermeiro durante os procedimentos.

A fala do participante acerca da exposição ao detergente e o que é preconizado pelas normas apresentaram diferença significativa, o que pode implicar um processamento ineficiente no tocante ao tempo desperdiçado e ao provável desgaste do artigo, tendo incidência direta sobre a eficiência do processo $^{11}$.

Após a lavagem, os materiais passam pela secagem completa, manual ou automatizada, utilizando-se material macio, que não libere fibras e de cor clara, para favorecer a identificação de possíveis sujidades, ou por meio de jato de ar comprimido nos materiais com lúmens, reentrâncias, válvulas, entre outros ${ }^{11}$. A maioria dos entrevistados relatou a etapa da secagem sem preocupação com a inspeção.

A investigação criteriosa da limpeza é um dos pontos críticos para que um produto seja reutilizado, pois resíduos podem impedir o contato do agente esterilizante e causar eventos adversos de ordem imunológica aos pacientes Além disso, os artigos que não estiverem íntegros nem funcionais devem ser trocados, para evitar danos ao paciente, a ampliação do tempo de duração da cirurgia, ou a insatisfação à equipe usuária ${ }^{11}$.

Estudo descritivo de casos múltiplos realizado em quatro hospitais de Salvador (BA) observou que os funcionários não realizavam sistematicamente a inspeção visual nem os testes de integridade e de funcionalidade necessários para prevenir riscos ligados às falhas de limpeza e possíveis alterações nas características dos produtos submetidos aos múltiplos ciclos de reprocessamento ${ }^{12}$. Esse fato assemelhou-se aos achados desta casuística, em que apenas dois profissionais apontaram a inspeção dos materiais como uma etapa primordial na qualidade da limpeza executada.

A maioria dos sujeitos mencionou a esterilização de forma sucinta, citando-a como parte do processo. Em contrapartida, apenas três entrevistados discorreram sobre seus tipos e suas especificidades, a exemplo do gás plasma de peróxido de hidrogênio e do vapor saturado sob pressão.

Alguns entrevistados demonstraram pouco conhecimento sobre as etapas do processamento dos materiais, apesar de terem nove anos de experiência, em média. Isso pode ser considerado um alerta para que sejam realizadas estratégias de transformação de realidades, como o desenvolvimento 
de ações educativas e estímulo à participação em eventos e discussões, entre outros, que visem à melhoria do conhecimento desses profissionais.

Pesquisa qualitativa com profissionais do CME demonstrou que os participantes não souberam descrever a etapa nem informar os tipos de esterilização. Elucidou-se que, além de conhecer cada etapa do processamento, é imprescindível o conhecimento de todo o processo, para garantir a ausência de contaminação dos materiais hospitalares e consequentes complicações ao paciente ${ }^{3}$.

O armazenamento é a parte final do processo. Nele, os produtos esterilizados devem ser estocados em local limpo e seco, sob proteção da luz solar direta, e submetidos à manipulação mínima, garantindo a sua integridade física e evitando contaminação ${ }^{3,11}$. Nessa visão, um dos participantes atribuiu importância ao controle do material nesse local.

Diante do processamento de materiais, o CME tem papel importante na prevenção e no controle de infecções. Para tanto, precisa de funcionamento adequado, de eficácia e de segurança no processo de trabalho, para proporcionar a qualidade necessária dos artigos esterilizados, contribuindo com a assistência ao paciente e à equipe cirúrgica ${ }^{3}$.

As atividades desenvolvidas no CME apresentam forte interferência no produto final durante a assistência. Qualquer falha no reprocessamento implica possível comprometimento da esterilidade dos produtos e possibilita aumento no risco de infecções em todos os procedimentos que são realizados nos pacientes, tais como cirurgias, curativos e punções venosas ${ }^{13}$. A unanimidade nas respostas demonstrou a importância de o processamento ser feito de forma correta para evitar possíveis complicações.

Em um estudo realizado em Teresina (PI), observouse que a equipe de enfermagem valorizava seu trabalho no CME e atribuía a assistência indireta ao paciente, salientando a necessidade de acompanhar os avanços tecnológicos e o aprimoramento dessas atividades para que a segurança do paciente fosse mantida ${ }^{14}$. Um dos participantes salientou a importância de avaliar a efetividade do processo de esterilização por meio de indicadores.

A prática da esterilização deve estar inserida em critérios preestabelecidos, ancorados pelas referências científicas e normatizações pertinentes, assegurando que os produtos reprocessados não sejam causadores de infecções ${ }^{3}$. Nesse sentido, os profissionais atuantes no CME devem ter responsabilidade ativa na prevenção e no controle das infecções hospitalares, adotar medidas com o propósito de causar a morte microbiana e garantir a segurança do processamento ${ }^{11}$.
No decorrer da pesquisa, os entrevistados expuseram as dificuldades encontradas na rotina de trabalho, apontando a falta de materiais fundamentais ao setor como responsável pelo mau funcionamento dele.

A gestão de materiais é um seguimento no qual se planeja, se executa e se controla o quantitativo de insumos por meio de estratégias eficazes e econômicas, no entanto tem sido motivo de preocupação nas instituições de saúde, principalmente no setor público, em razão de orçamentos restritos, que exigem mais controle do consumo e dos gastos para que funcionários e pacientes não sejam privados do material necessário, nem haja interferência na qualidade do processamento ${ }^{15}$.

Um estudo buscou avaliar os riscos ocupacionais no ambiente do CME e demonstrou que a maioria dos profissionais afirmou que EPIs devem ser usados e cuidados para evitar acidentes são básicos ${ }^{16}$. Nesta casuística, encontrou-se semelhante preocupação, ainda que tenha sido destacada a falta desses equipamentos no setor, por conta da importância que os profissionais lhes atribuem na prevenção de acidentes de trabalho, apresentando-se como um fator positivo desta pesquisa.

Durante o desenvolvimento de suas atividades laborais, os trabalhadores devem estar atentos aos riscos ocupacionais aos quais estão expostos. O biológico é o mais comum, uma vez que os funcionários entram em contato continuamente com sangue e outros fluidos corpóreos ao receberem o produto contaminado. Com a finalidade de proteger o funcionário dos riscos suscetíveis de ameaçar sua segurança e saúde, é recomendado e obrigatório o uso dos EPIs, que são todos os dispositivos de uso individual utilizados pelo indivíduo no exercício de sua função ${ }^{17}$.

Os EPIs, como gorro, óculos, máscara, luvas grossas de borracha, avental impermeável e sapatos fechados e emborrachados, são ferramentas de trabalho que visam proteger a saúde do trabalhador e amenizar os riscos aos quais estão expostos. Portanto, cabem às empresas o fornecimento correto e a realização de testes e treinamentos quanto ao uso, bem como ouvir sugestões e críticas ${ }^{17}$. Por outro lado, percebeuse certa resistência dos profissionais quanto ao uso dos EPIs.

Autores trouxeram a hipótese de que os trabalhadores com mais tempo de serviço e experiência poderiam se sentir mais seguros e acabar se descuidando de determinadas precauções, por confiar em demasiado nas suas destrezas, causando, assim, ocasionalmente acidentes ${ }^{18}$. A população do estudo tinha, em média, 22,6 anos de experiência profissional, o que pode ser um alerta aos demais serviços.

Em pesquisa realizada em seis hospitais de Londrina (PR) sobre o uso de EPIs no CME, foram identificados sentimentos 
negativos em relação ao seu uso, tais como incômodo, dificuldade na utilização, calor, entre outros, mas os profissionais revelaram que faziam uso dos utensílios por ser norma da instituição; contudo, se pudessem escolher, não os usariam $^{17}$. Essas opiniões convergem com os discursos dos trabalhadores referentes à adesão aos EPIs.

Outro fator que interfere na qualidade dos serviços de esterilização é a quantidade de trabalhadores existentes, que é, frequentemente, insuficiente para a demanda do setor. Isso pode ocorrer pela falha no dimensionamento dos profissionais de enfermagem, afetando, assim, o funcionamento operacional na sua prática diária ${ }^{1,19}$.

Muitas vezes, o CME torna-se um espaço esquecido pelos gestores, ao considerar que são encaminhados para sua lotação os profissionais de enfermagem com problemas físicos ou psicológicos, com idades avançadas, readaptados, em pré-aposentadoria ou por inadequação de relacionamento em diferentes áreas da assistência, incapazes, portanto, de desempenhar atividades assistenciais diretas ${ }^{19}$.

Ao designar profissionais com problemas de saúde, de relacionamento e/ou com defasagem de conhecimentos para um setor de alta complexidade técnica como o CME, comete-se um erro, pois, para garantir a qualidade dos serviços e da assistência prestados nas unidades de saúde, os trabalhadores precisam possuir perfil satisfatório e capacitação teórico-prática ${ }^{1}$.

O enfermeiro responsável pelo CME precisa estabelecer aliados com os serviços de educação continuada e traçar estratégias de enfrentamento diante da escassez de recursos humanos, como o gerenciamento das atividades a serem desenvolvidas, prevendo e organizando as prioridades, sem colocar em risco a segurança ou a qualidade do processamento ${ }^{19}$.

Evidenciou-se a necessidade de ações em educação e saúde, quanto aos procedimentos realizados no processamento de produtos, uma vez que os profissionais afirmaram que a falta de capacitação é um limitante na superação das dificuldades diárias.

Por ser um setor de diversas peculiaridades para manter a equipe em sincronia, o desenvolvimento de programas contínuos de treinamento e aperfeiçoamento dos trabalhadores pela educação permanente poderia ser útil para a prevenção dessas situações ${ }^{20}$. Diante desses aspectos, associar a teoria à prática proporcionará aos funcionários melhor assimilação e raciocínio crítico sobre a necessidade e a importância dos pequenos cuidados com os materiais ${ }^{9}$.

As pessoas, quando motivadas, desenvolvem o seu trabalho de maneira equilibrada e produtiva ${ }^{9}$. De acordo com as narrativas, quando ocorriam cursos de educação permanente associados à remuneração extra nos proventos, os trabalhadores sentiam-se estimulados a participar, sendo esse aspecto da instituição considerado positivo.

Em estudo realizado em São Paulo (SP), a maioria dos enfermeiros reunia seus funcionários para orientá-los, geralmente quando eram adquiridos novos equipamentos pela instituição 9 , o que convergiu com os achados nas falas dos respondentes ao explicarem a rotina com a chegada de um novo produto químico ou máquina. A despeito da importância do enfermeiro como gerenciador dessas atividades, apenas um dos respondentes destacou o papel desse profissional como educador.

Assim, é importante refletir que, para o enfermeiro alcançar seu reconhecimento profissional, deve transformar o espaço de trabalho em um ambiente agradável, no qual toda a equipe possa se sentir acolhida, com seu olhar voltado para a valorização do cuidado e para a humanização, além de estar fundamentado em pesquisas científicas e ações efetivas de educação permanente em saúde ${ }^{3}$.

\section{CONSIDERAÇÕES FINAIS}

O CME é um setor de vital importância na prevenção e no controle das infecções relacionadas à assistência à saúde, pelo funcionamento compatível às suas diretrizes, para que todas as etapas sejam realizadas com eficácia e segurança, pois seu serviço influencia na qualidade da assistência indireta prestada ao paciente.

Assim, torna-se necessário implantar programas de educação em saúde que abranjam todos os profissionais da área, para que se busquem transformações no método de trabalho, por meio de sensibilização, envolvimento, compartilhamento e aplicação do conhecimento científico nas práticas diárias.

No tocante ao processo de esterilização do material cirúrgico, notou-se que alguns profissionais mostraram ter pouco conhecimento sobre as etapas envolvidas no reprocessamento. Percebeu-se que surgem dúvidas e inseguranças durante a prática, assim como ocorre a falta de materiais necessários, entre outras dificuldades, o que compromete seriamente a eficácia da esterilização.

O profissional que desenvolve suas atividades no CME tem de estar apto para acompanhar o desenvolvimento tecnológico que aperfeiçoa o serviço. Portanto, é fundamental a participação do enfermeiro na qualificação do processo laboral de esterilização de materiais e na identificação das necessidades de sua equipe, para garantir a eficiência dos processos 
e contribuir para a prevenção de infecções. Dessa maneira, a constatação das dificuldades propõe mobilizar os sujeitos a implementarem mudanças em seu processo de trabalho, a fim de superar tais fragilidades, além da necessidade de realização de pesquisas futuras que complementem os dados obtidos aqui.
No decorrer do estudo, ocorreram algumas limitações, como a coleta de dados durante o horário de trabalho dos funcionários. Isso pode ter comprometido, em parte, as falas dos participantes, já que o CME é um ambiente de constantes atividades, e o profissional pode ter respondido brevemente algumas indagações para retomar rapidamente as suas atividades.

\section{REFERÊNCIAS}

1. Ascari RA, Vidori J, Moretti CA, Perin EMF, Silva OM, Buss E. 0 processo de esterilização de materiais em serviços de saúde: uma revisão integrativa. Braz J Surgery Res [Internet]. 2013 [citado em out. 2016];4(2):33-8. Disponível em: http://www.mastereditora.com. br/periodico/20130831_181149.pdf

2. Brasil. Ministério da Saúde. Agência Nacional de Vigilância Sanitária. Resolução da Diretoria Colegiada RDC n 15, de 15 de março de 2012. Dispõe sobre requisitos de boas práticas para o processamento de produtos para saúde e dá outras providências. Diário Oficial da União; 2012.

3. Ouriques MC, Machado ME. Enfermagem no processo de esterilização de materiais. Texto Contexto Enferm. 2013;22(3):695-703. http:// dx.doi.org/10.1590/S0104-07072013000300016

4. Carvalho JDC. Interface do processamento de materiais na central de materiais e esterilização e a segurança do paciente em um hospital do sul do país [dissertação] [Internet]. Florianópolis: Universidade Federal de Santa Catarina; 2012 [citado em nov. 2016]. Disponível em: http://repositorio.ufsc.br/xmlui/handle/123456789/99339

5. Gil RF, Camelo HS, Laus AM. Atividades do enfermeiro de centro de material e esterilização em instituições hospitalares. Texto Contexto Enferm. 2013;22(4):927-34. http://dx.doi.org/10.1590/S0104-07072013000400008

6. Padoveze MC, Figueiredo RM, Pelaes CES, Otrenti E. Necessidades de aprendizagem de enfermeiros sobre processos de esterilização. Rev SOBECC [Internet]. 20132012 [citado em dez. 2016];18(3):239. Disponível em: http://www.sobecc.org.br/arquivos/artigos/2014/ pdfs/revisao-de-leitura/Ano18_n3_\%20jul_set2013-3.pdf

7. Minayo MC. 0 desafio do conhecimento: pesquisa qualitativa em saúde. São Paulo: Hucitec; 2004.

8. Bardin L. Análise de conteúdo. São Paulo: Edições 70; 2011.

9. Souza MCB, Ceribelli MIPF. Enfermagemno centro de material esterilizado: a prática da educação continuada. Rev Latino-Am Enferm. 2004;12(5):76774. http://dx.doi.org/10.1590/S0104-11692004000500010

10. Brasil. Ministério da Saúde. Agência Nacional de Vigilância Sanitária. Informe Técnico n01/09. Princípios básicos para limpeza de instrumental cirúrgico em serviços de saúde unidade de investigação e prevenção das infecções e dos eventos adversos, UIPEA. Gerência Geral de Tecnologia em Serviços de Saúde [Internet]. Brasília: Ministério da Saúde; 2009 [citado 28 fev. 2017]. Disponível em: http://www.anvisa. gov.br/servicosaude/controle/alertas/2009/informe_tecnico_1.pdf
11. Associação Brasileira de Enfermeiros de Centro Cirúrgico, Recuperação Anestésica e Centro de Material e Esterilização (SOBECC). Diretrizes de práticas em enfermagem cirúrgica e processamento de produtos para a saúde. $7^{\text {7a }}$ ed. São Paulo: SOBECC; 2017.

12. Costa EAM, Costa EA. Risco e segurança sanitária: análise do reprocessamento de produtos médicos em hospitais de Salvador BA. Rev Saúde Pública. 2012;46(5):800-7. http://dx.doi.org/10.1590/ S0034-89102012000500006

13. Anjos MAM, Oliveira JC. As percepções dos profissionais de enfermagem da central de material e esterilização: uma reflexão sobre a cultura organizacional. Rev ACRED [Internet]. 2016 [citado em jan. 2017];6(11):1-9. Disponível em: https://dialnet.unirioja.es/ servlet/articulo?codigo $=5602109$

14. Silva PSC, Santos MV, Costa CRM. Atuação da enfermagem na central de material e esterilização em um hospital de Teresina. Rev Interd. 2013;6(3):45-51.

15. Garcia SD, Haddad MCL, Dellaroza SG, Costa DB, Miranda JM. Gestão de material médico-hospitalar e o processo de trabalho em um hospital público. Rev Bras Enferm. 2012;65(2):339-46. http:// dx.doi.org/10.1590/S0034-71672012000200021

16. Espíndola MCG, Fontana RT. Riscos ocupacionais e mecanismos de autocuidado do trabalhador de um centro de material e esterilização. Rev Gaúcha Enferm. 2012;33(1):116-23. http://dx.doi.org/10.1590/ S1983-14472012000100016

17. Ribeiro RP, Vianna LAC. Uso dos equipamentos de proteção individual entre trabalhadores das centrais de material e esterilização. Ciênc Cuid Saúde. 2012;11(Supl.):199-203. http://dx.doi.org/10.4025/ cienccuidsaude.v11i5.17076

18. Aquino JM, Barros LP, Brito AS, Ferreira EB, Medeiros SEG, Santos ER. Centro de material e esterilização: acidentes de trabalho e riscos ocupacionais. Rev SOBECC. 2014;19(3):148-54. http://dx.doi. org/10.4322/sobecc.2014.023

19. Athanázio AR, Cordeiro BC. Educação permanente a trabalhadores da central de material e esterilização. RevEnfermUFPE. 2015;9(Supl. 6):875861. https://doi.org/10.5205/1981-8963-v9i6a10657p8758-8761-2015

20. Pezzi MCS, Leite JL. Investigação em central de material e esterilização utilizando a teoria fundamentada em dados. Rev Bras Enferm. 2010;63(3):391-6. http://dx.doi.org/10.1590/ S0034-71672010000300007 